\title{
Isothermal adsorption of polyampholytes on charged nanopatterned surfaces
}

Cite as: J. Chem. Phys. 151, 084101 (2019); https://doi.org/10.1063/1.5115404

Submitted: 17 June 2019 . Accepted: 01 August 2019 . Published Online: 22 August 2019

Amin Bakhshandeh (D, Alexandre P. dos Santos, Alexandre Diehl, and Yan Levin (i)

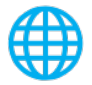

\section{ARTICLES YOU MAY BE INTERESTED IN}

Compressing physics with an autoencoder: Creating an atomic species representation to improve machine learning models in the chemical sciences

The Journal of Chemical Physics 151, 084103 (2019); https://doi.org/10.1063/1.5108803

Relativistic theory of electron-nucleus-radiation coupled dynamics in molecules:

Wavepacket approach

The Journal of Chemical Physics 151, 084102 (2019); https://doi.org/10.1063/1.5109272

Interaction between random heterogeneously charged surfaces in an electrolyte solution

The Journal of Chemical Physics 142, 194707 (2015); https://doi.org/10.1063/1.4921410

\section{Lock-in Amplifiers up to $600 \mathrm{MHz}$}
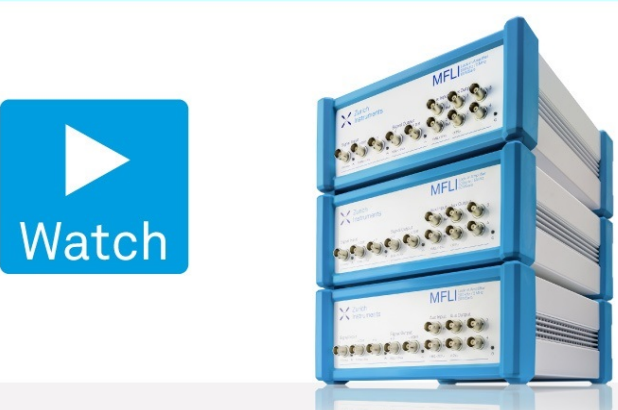

J. Chem. Phys. 151, 084101 (2019); https://doi.org/10.1063/1.5115404 


\title{
Isothermal adsorption of polyampholytes on charged nanopatterned surfaces
}

\author{
Cite as: J. Chem. Phys. 151, 084101 (2019); doi: 10.1063/1.5115404 \\ Submitted: 17 June 2019 - Accepted: 1 August 2019 • \\ Published Online: 22 August 2019
}

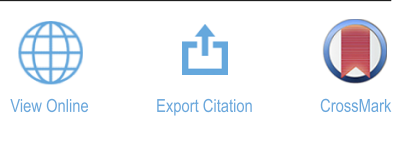

Amin Bakhshandeh, ${ }^{1, a)}$ (D) Alexandre P. dos Santos, ${ }^{2, b)}$ Alexandre Diehl, ${ }^{3, c)}$ and Yan Levin ${ }^{2, d)}$ (D)

\begin{abstract}
AFFILIATIONS Caixa Postal 354, CEP 96010-900 Pelotas, RS, Brazil CEP 96010-900 Pelotas, RS, Brazil

\author{
a) Electronic mail: amin.bakhshandeh@ufrgs.br \\ b) Electronic mail: alexandre.pereira@ufrgs.br \\ ${ }^{c}$ Electronic mail: diehl@ufpel.edu.br \\ d) Electronic mail: levin@if.ufrgs.br
}

'Programa de Pós-Graduação em Física, Instituto de Física e Matemática, Universidade Federal de Pelotas,

${ }^{2}$ Instituto de Física, Universidade Federal do Rio Grande do Sul, Caixa Postal 15051, CEP 91501-970, Porto Alegre, RS, Brazil

${ }^{3}$ Departamento de Física, Instituto de Física e Matemática, Universidade Federal de Pelotas, Caixa Postal 354,

\begin{abstract}
We investigate the adsorption of neutral polyampholytes on charged nanopatterned surfaces. The surfaces have charged domains but are overall neutral. To perform efficient simulations, we use an approach which combines the explicit form of the interaction potential between the polyampholyte monomers and the surface with a 3d Ewald summation method. We observe that the amount of adsorption and the structure of the adsorbed polyampholytes depend strongly on the surface pattern, the relative size of the surface domains, and the charge distribution along the polyampholyte backbone.
\end{abstract}

Published under license by AIP Publishing. https://doi.org/10.1063/1.5115404

\section{INTRODUCTION}

Polymers are of great technological importance. Polyampholytes are the types of polymers whose monomers become ionized in polar solvents. This makes polyampholytes very soluble in water with a strong affinity for charged surfaces. ${ }^{1-4}$ Polyampholytes have wide applications in biomedicine, ${ }^{5}$ water purification, ${ }^{6}$ food technology, and drag reduction. ${ }^{8}$ Proteins are considered natural polyampholytes, ${ }^{9}$ making understanding of these polymers very important for biology and biophysics. Laboratory syntheses of polyampholytes can be performed using radical and emulsion polymerization. ${ }^{10,1}$ Polyampholytes can also be synthesized by copolymerization of an acidic and a basic monomer, such as acrylic acid and vinylpyridine. ${ }^{12}$ Depending on $\mathrm{pH}$, polyampholytes can be overall charge neutral-if the number of positive and negative monomers is the same-or have a finite net charge. ${ }^{10}$ The $3 \mathrm{~d}$ configuration of polyampholyte molecules is governed by the competition between electrostatic and entropic forces. Inside an aqueous solution, neutral random polyampholytes exist in a dilute globular form; ${ }^{13-15}$ on the other hand, polyampholytes with an alternating sequence of positive and negative monomers are in a coil state, with the endto-end distance scaling as $R \sim N^{3 / 5}$, where $N$ is the number of monomers. ${ }^{16,17}$

One way to evaluate physical and chemical properties of materials is through their interaction with surfaces. For example, in catalysis, the molecules must adsorb to the surface of the catalyst. The rate of reaction will depend on the rate of adsorption and adherence. Mean field theories were developed to study polyampholytes in bulk ${ }^{14,18,19}$ and interacting with uniformly charged surfaces. ${ }^{20-24}$ On the other hand, there is very little literature ${ }^{25,26}$ on the interaction of polyampholytes with heterogeneously charged surfaces. ${ }^{27-2}$ Nanopatterned surfaces are particularly interesting due to the complex behavior of ions near the charged patches. ${ }^{30}$ Furthermore, the periodic pattern of the surface becomes imprinted on the electrolyte solution through the renormalization of the Debye length, which controls the interactions between the charged species and 
the nanopatterned wall. ${ }^{31,32}$ Lack of continuous symmetry and presence of strong ionic interactions between charged patches and the monomers of polyampholyte makes the problem very difficult to study using analytical methods. ${ }^{33-36}$ On the other hand, computer simulations provide a direct way to explore both the adsorption and the structure of the polyampholytes near a nanopatterned surface. Recently, we have introduced a Monte Carlo (MC) method which allows one to efficiently simulate nanopatterned charged surfaces inside an electrolyte solution. ${ }^{31}$ In this approach, the long range Coulomb interaction between the ions is treated using a modified $3 \mathrm{~d}$ Ewald summation method. ${ }^{37}$ On the other hand, the interaction with the surfaces is taken into account through the exact analytical solution of the Poisson equation. ${ }^{31}$ In the present work, we will use this Monte Carlo method to explore the adsorption of polyampholytes on nanopatterned charged surfaces. Our aim is to understand the structure of polyampholytes inside the solution and the degree of their adsorption to the nanopatterned surface as a function of charge distribution on the surface and on the polyampholyte backbone.

This paper is organized as follows: In Sec. II, we explain the model and present the simulation method. In Sec. III, we summarize our results. In Sec. IV, the conclusions are presented.

\section{THE MODEL AND SIMULATION DETAILS}

The overall neutral nanopatterned surface is described by a sinusoidal charge density,

$$
\sigma(x, y)=\sigma_{0} \sin \left(k_{x} x\right) \sin \left(k_{y} y+\varphi_{y}\right)
$$

where $\sigma_{0}$ is the amplitude and $\varphi_{y}=\pi / 2$ is the phase, $k_{x}=2 \pi n_{x} / L_{x}$, $k_{y}=2 \pi n_{y} / L_{y}$, with $L_{x}$ and $L_{y}$ periods of charge density variation in $x$ and $y$ directions, respectively, and $n_{x, y}$ are integers. In the present work, we considered a striped domain of various periodicity: $\left(n_{x}=1\right.$, $\left.n_{y}=0\right),\left(n_{x}=2, n_{y}=0\right),\left(n_{x}=3, n_{y}=0\right)$, and $\left(n_{x}=4, n_{y}=0\right)$, which are represented in Fig. 1 . We fix $\sigma_{0}=0.1 \mathrm{C} / \mathrm{m}^{2}$, except in the last figure. It can be shown that the electrostatic potential at position $\boldsymbol{r}=(x, y, z)$ produced by this charge density is ${ }^{3}$

$$
\Phi_{1}(\boldsymbol{r})=\frac{2 \pi \sigma_{0}}{\epsilon_{w} \alpha} \sin \left(k_{x} x\right) \sin \left(k_{y} y+\varphi_{y}\right) e^{-\alpha|z|},
$$

where $\alpha=\sqrt{k_{x}^{2}+k_{y}^{2}}$.

The present system consists of two flat surfaces of dimensions $L_{x}$ and $L_{y}$, located at $z=0$ and $z=L$ enclosing the polyampholyte solution. The surfaces are identical. The electrostatic potential produced by the two surfaces can be obtained by the superposition,

$$
\Phi(\boldsymbol{r})=\frac{2 \pi \sigma_{0}}{\epsilon_{w} \alpha} \sin \left(k_{x} x\right) \sin \left(k_{y} y+\varphi_{y}\right)\left(e^{-\alpha|z|}+e^{-\alpha|z-L|}\right) .
$$

We use two identical bounding surfaces in order to improve the statistics for calculating the adsorption isotherms.

The polyampholytes are composed of $N_{m}$ spherical monomers of radius $2 \AA$ and unit charge located at the center. In this work, we (a)

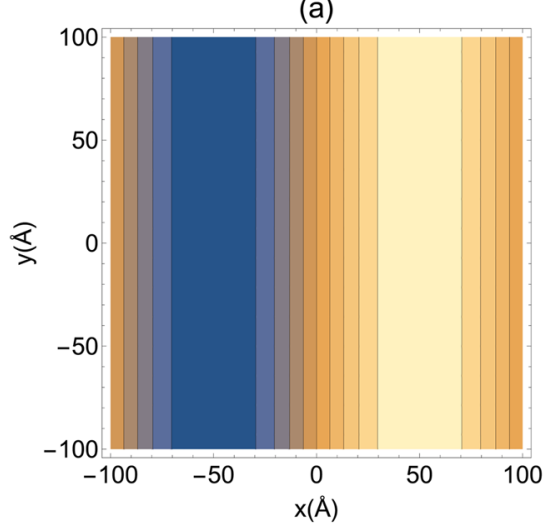

(c)

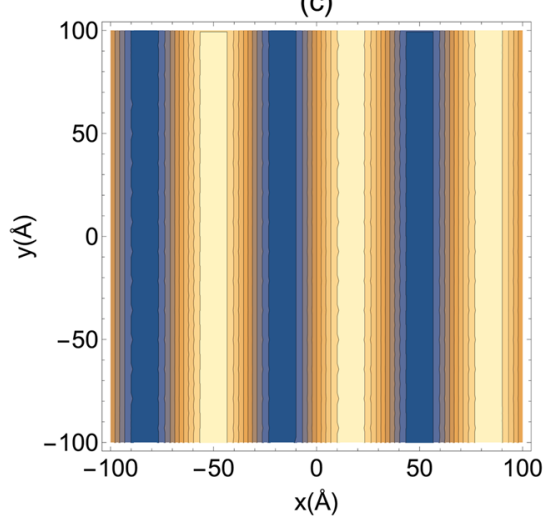

(b)

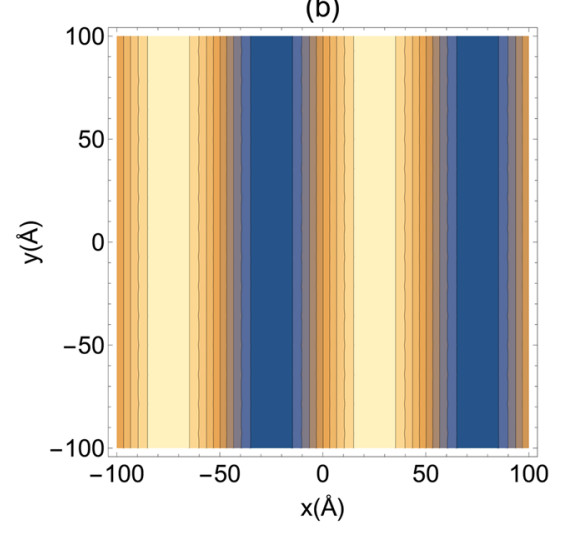

(d)

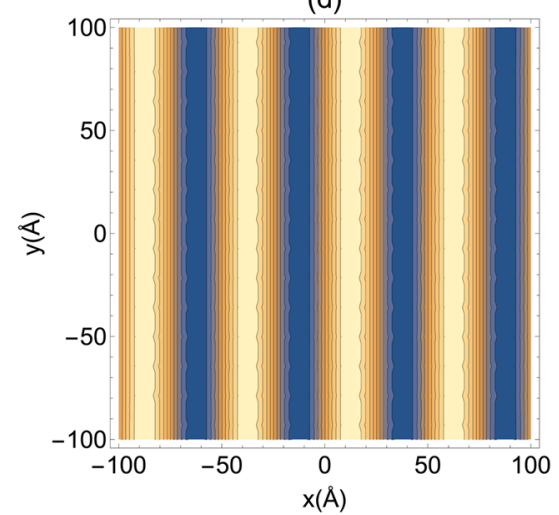

FIG. 1. Four charge patterns, corresponding to striped configurations with different wavelengths, are investigated in the present study with $L_{x}=L_{y}=200 \AA$. (a) $n_{x}=1, n_{y}=0$. (b) $n_{x}=2$, $n_{y}=0$. (c) $n_{x}=3, n_{y}=0$. (d) $n_{x}=4$, $n_{y}=0$. Dark and light regions represent oppositely charged domains. 
will explore the behavior of polyampholytes with different periodicities. For 1-1 periodicity, the charges of the monomers are repeated as $-e, e, \ldots$ and for $2-2$ the charges are $-e,-e,+e,+e, \ldots$ while for 3-3 they are $-e,-e,-e,+e,+e,+e, \ldots$. The adjacent monomers that compose a chain interact via a simple parabolic potential which approximates the stretching of molecular bonds ${ }^{36}$

$$
U_{b}(r)=\frac{A}{2}\left(r-r_{0}\right)^{2},
$$

where $A=0.97 k_{B} T, r$ is the distance between adjacent monomers, and $r_{0}=5 \AA$. The solvent, water, is assumed to be a uniform dielectric continuum of permittivity $\epsilon_{w}$. The Bjerrum length is defined as $\lambda_{B}=e^{2} / k_{B} T \epsilon_{w}$, where $e, k_{B}$, and $T$ are the elementary charge, the Boltzmann constant, and the absolute temperature, respectively. For water at room temperature, the Bjerrum length is $\lambda_{B}=7.2 \AA$. The simulations are performed using the Metropolis algorithm ${ }^{38,39}$ with $10^{7}$ MC steps for equilibration. Each sample is obtained with 300 trial movements per particle. The macromolecules can perform rotation, translation, and reptation. Moreover, monomers can attempt short displacements. To account for the electrostatic interactions between the monomers, we use the modified 3d Ewald summation method. ${ }^{37,40}$

The total energy of the system is

$$
\begin{aligned}
U= & \sum_{\boldsymbol{k} \neq \boldsymbol{0}}^{\infty} \frac{2 \pi}{\epsilon_{w} V|\boldsymbol{k}|^{2}} \exp \left[-\frac{|\boldsymbol{k}|^{2}}{4 \kappa_{e}^{2}}\right]\left[A(\boldsymbol{k})^{2}+B(\boldsymbol{k})^{2}\right] \\
& +\frac{2 \pi}{\epsilon_{w} V}\left(M_{z}^{2}-Q_{t} G_{z}\right)+\frac{1}{2} \sum_{i \neq j}^{N} q_{i} q_{j} \frac{\operatorname{erfc}\left(\kappa_{e}\left|\boldsymbol{r}_{i}-\boldsymbol{r}_{j}\right|\right)}{\epsilon_{w}\left|\boldsymbol{r}_{i}-\boldsymbol{r}_{j}\right|} \\
& +\sum_{i=1}^{N} q_{i} \Phi\left(\boldsymbol{r}_{i}\right)+\sum_{a d j .} U_{b}\left(\left|\boldsymbol{r}_{i}-\boldsymbol{r}_{j}\right|\right),
\end{aligned}
$$

where

$$
\begin{aligned}
A(\boldsymbol{k}) & =\sum_{i=1}^{N} q_{i} \cos \left(\boldsymbol{k} \cdot \boldsymbol{r}_{i}\right), \\
B(\boldsymbol{k}) & =-\sum_{i=1}^{N} q_{i} \sin \left(\boldsymbol{k} \cdot \boldsymbol{r}_{i}\right), \\
M_{z} & =\sum_{i=1}^{N} q_{i} z_{i}, \\
G_{z} & =\sum_{i=1}^{N} q_{i} z_{i}^{2}, \\
Q_{t} & =\sum_{i=1}^{N} q_{i} .
\end{aligned}
$$

$N$ is the total number of monomers, $\boldsymbol{r}_{i}=\left(x_{i}, y_{i}, z_{i}\right)$ is the position of monomer $i$, and $V=L_{x} L_{y} L_{z}$ is the volume of the simulation cell, with $L_{z}=3 L_{x}$. Note that the simulation cell also includes a vacuum region outside the two surfaces. The $\boldsymbol{k}$ vectors are defined as $\boldsymbol{k}=\left(\frac{2 \pi}{L_{x}} n_{x}, \frac{2 \pi}{L_{y}} n_{y}, \frac{2 \pi}{L_{z}} n_{z}\right)$, where $n^{\prime} s$ are integers. Around 500 vectors are used in the calculations. The damping parameter is $\kappa_{e}=5 / L_{x}$. The symbol adj. below the last sum in Eq. (5) signifies that the sum is over adjacent monomers in the macromolecules. The overlap between particles $i$ and $j$ is always considered a rejected MC move. In the present system, the net charge between the surfaces is $Q_{t}=0$.

\section{RESULTS}

To explore the presence of finite size effects, we study two system sizes: $L_{x}=L_{y}=200 \AA$ with $n_{x}=1$ and $L_{x}=L_{y}=400 \AA$ with $n_{x}=2$. In both cases, the separation between surfaces is $L=300 \AA$. Note that the wavelength of the surface charge density variation for these two systems is identical. Therefore, we expect identical adsorption, if finite size effects are negligible. The polyampholytes have 6 monomers. The number of polymers is adjusted to produce the same bulk concentration, $14 \mathrm{mM}$, inside the two simulation cells. We find that the polymer density profiles for the two systems are identical [see Fig. 2(a)]. This means that the cell with $L_{x}=L_{y}=200 \AA$ is sufficiently large to avoid finite size effects. The adsorption is due to the electrostatic interaction. To see this more clearly, we consider 10 polyampholytes of 18 monomers each, with periodicity $1-1$, in two different systems: one with a charged patterned surfaces and the other with a nonpatterned neutral hard wall. As can be seen from Fig. 2, while polyampholytes are adsorbed to the patterned surface, they are repelled from the neutral hard wall. In the latter case, the polyampholyte depletion is due to the steric repulsion, which prevents the polymer coils from approaching the wall closer than their characteristic radius of gyration.

For many chemical and physical processes, it is important to know adsorption isotherms. The adsorption per unit of area in the present geometry is given by

$$
\Gamma=\frac{1}{2} \int_{0}^{L}\left[\rho(z)-\rho_{b}\right] d z,
$$

where $\rho(z)$ and $\rho_{b}$ are the local and bulk concentrations. We first consider polyampholytes of 18 monomers with periodicity $1-1$. The concentration profiles are calculated using the center of mass of the macromolecules. In Fig. 3, we show the concentration profile of polyampholytes for the surfaces with charge patterns $n_{x}=1$ and $n_{y}=0$. The concentration of macromolecules near the surface is higher than in the bulk, showing that the macromolecules are being
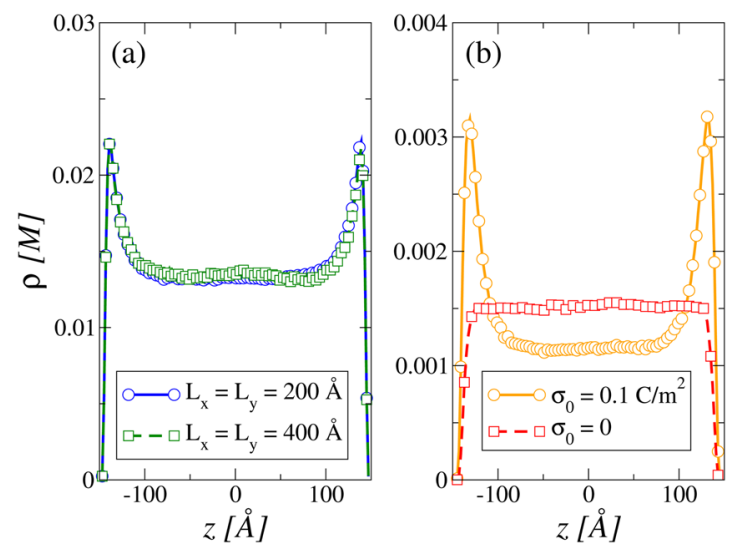

FIG. 2. (a) Center of mass density profile of 6-monomer polyampholytes with periodicity 1-1 for different system sizes. The bulk concentration of polyampholyte is $14 \mathrm{mM}$. The system sizes are $L_{x}=L_{y}=200 \AA$ with $n_{x}=1$ and $L_{x}=L_{y}=400$ $\AA$ with $n_{x}=2$, represented by circles and squares, respectively $\left(n_{y}=0\right)$. (b) Comparison between polyampholyte center of mass density profile for charged nanopatterned and uncharged surfaces. The system sizes are $L_{x}=L_{y}=200 \AA$ with $n_{x}=1, n_{y}=0$. 


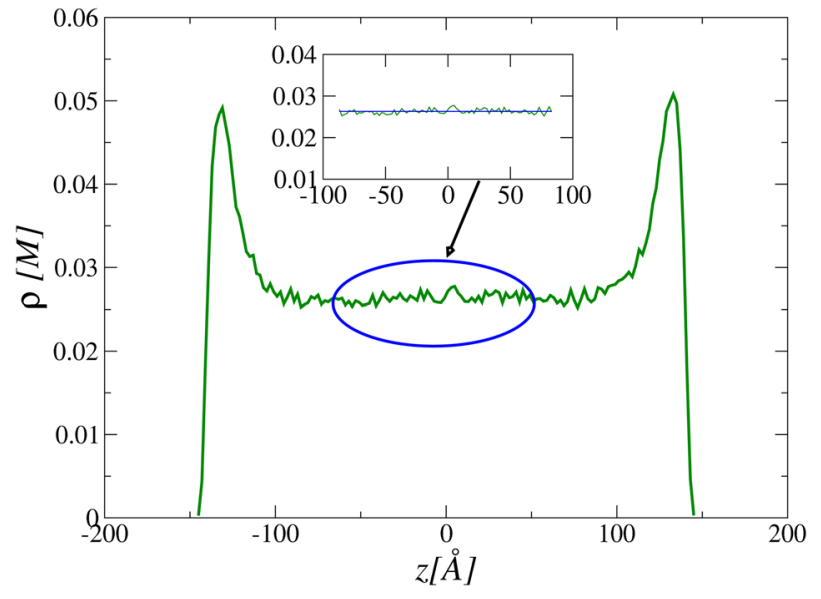

FIG. 3. Center of mass concentration profile of 18-monomer polyampholytes with periodicity 1-1 for $n_{x}=1$ and $n_{y}=0$. The bulk concentration is obtained by taking the average in the middle of the simulation cell.

adsorbed to the surface. Figure 3 shows how the bulk concentration $\rho_{b}$ can be obtained from the density profile. Once the bulk concentration of the polyampholytes is determined, the adsorption isotherm can be easily calculated as

$$
\Gamma=\frac{N_{p}}{2 L_{x} L_{y}}-\frac{\rho_{b} L}{2},
$$

where $N_{p}$ is the total number of macromolecules inside the simulation box. In Fig. 4, we show $\Gamma$ for patterns with $n_{y}=0$ and $n_{x}=1$, $n_{x}=2, n_{x}=3, n_{x}=4$, and also for $n_{x}=5$. As can be seen, by increasing $n_{x}, \Gamma$ rapidly decreases and inverts sign. The negative adsorption occurs when there is a sufficiently large depletion region between the polyampholyte center of mass and the wall. We conclude that the adsorption is strongly correlated with the width of charged domains. For $n_{x}=1$ and $n_{x}=2$, the adsorption is positive, showing that the electrostatic attraction dominates. For other cases, the adsorption is

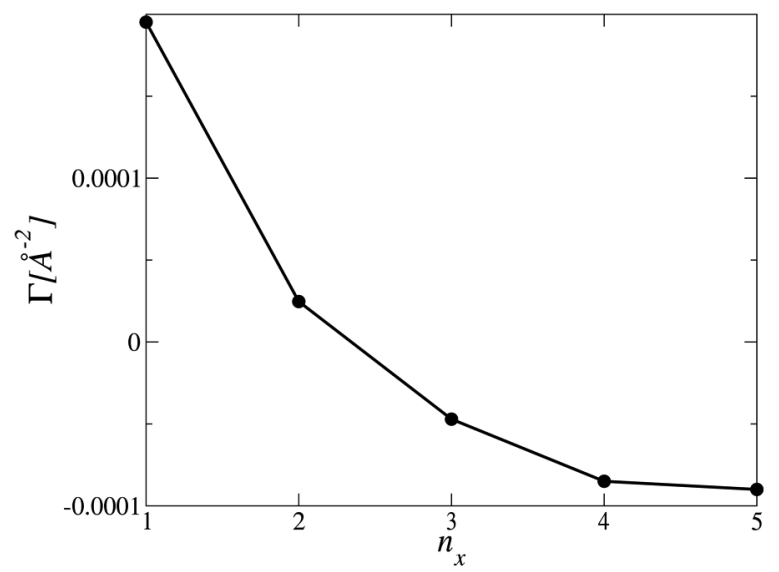

FIG. 4. Adsorption $\Gamma$ for different $n_{x}$. The bulk concentration is adjusted to $\approx 26 \mathrm{mM}$. The 18-monomer polyampholytes have periodicity $1-1$.

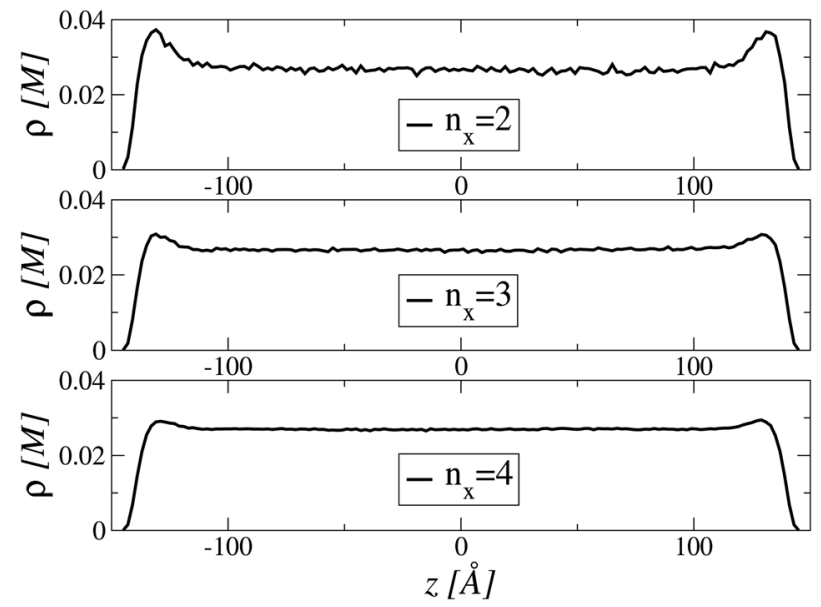

FIG. 5. Center of mass concentration profiles of 18-monomer polyampholytes with periodicity $1-1$ for different surface configurations. $n_{y}=0$. From top to bottom, $n_{x}=2, n_{x}=3$, and $n_{x}=4$.

negative, revealing the dominance of entropic repulsive interactions. In Fig. 5, we plot the polyampholyte density profile for patterns $n_{x}=2, n_{x}=3$, and $n_{x}=4$. The peak near the surface decreases with increasing $n_{x}$, consistent with the $\Gamma$ calculations.

In order to better understand the structure of polyampholytes near the surfaces, in Fig. 6, we show the concentration of positive and negative monomers for the same parameters as used in Fig. 3. As expected, the monomers tend to cluster near the oppositely charged

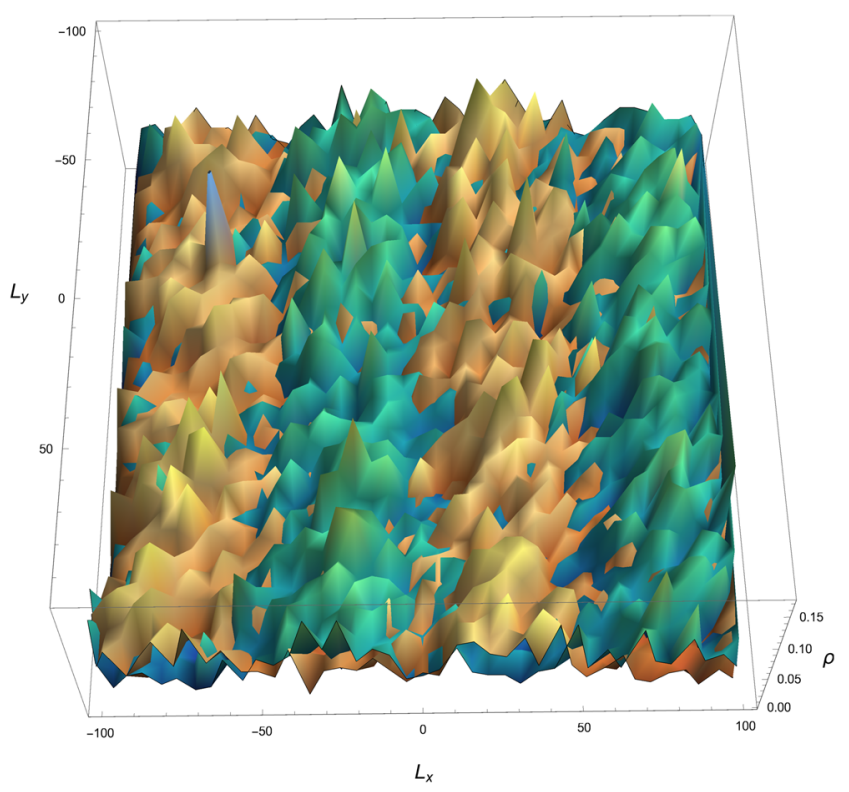

FIG. 6. Concentration of positive and negative monomers of 18-monomer polyampholytes with periodicity 1-1 within distance $\Delta z=6 \AA$ of a surface for $n_{x}=2$ and $n_{y}=0$. The colors represent positive and negative charges. 
domains. However, it is difficult to get a clear picture of the conformational structure of individual polyampholytes from this graph. In order to obtain an additional insight into the $3 \mathrm{~d}$ conformation of the macromolecules, we calculate the rms head-to-tail vector for the polyampholytes with the center of mass at position $z$. The rms components of the head-to-tail vector are defined as

$$
\begin{aligned}
& \Delta x(z)=\sqrt{\left\langle\left(x^{h}-x^{t}\right)^{2}\right\rangle_{z}}, \\
& \Delta y(z)=\sqrt{\left\langle\left(y^{h}-y^{t}\right)^{2}\right\rangle_{z}}, \\
& \Delta z(z)=\sqrt{\left\langle\left(z^{h}-z^{t}\right)^{2}\right\rangle_{z}},
\end{aligned}
$$

where $\left(x^{h}, y^{h}, z^{h}\right)$ and $\left(x^{t}, y^{t}, z^{t}\right)$ are the positions of the first and last monomers of the polyampholyte with the center of mass located at the position $z$. The brackets correspond to the sample average after the system has reached equilibrium. In Fig. 7, we plot the rms components for the patterns with $n_{x}=1$ and $n_{x}=2$. The figure shows that in the bulk, polyampholytes have a spherical coil shape. On the other hand, near the surface, the coil becomes stretched-attaining an ellipsoidal conformation with elongation along the $x$-axis, perpendicular to the orientation of surface charged stripes-see Fig. 1. The preference for the elongation along the $x$-axis can be understood purely on energetic grounds. We find that the electrostatic energy of the configuration in which the oligomer is adsorbed along a charged stripe [see Fig. 7(b)] is larger than when it is adsorbed transversally to the charged domains [Fig. 7(a)].

Neutral polyampholytes can have different periodicities of charged monomers along the backbone. In Fig. 8, we plot the

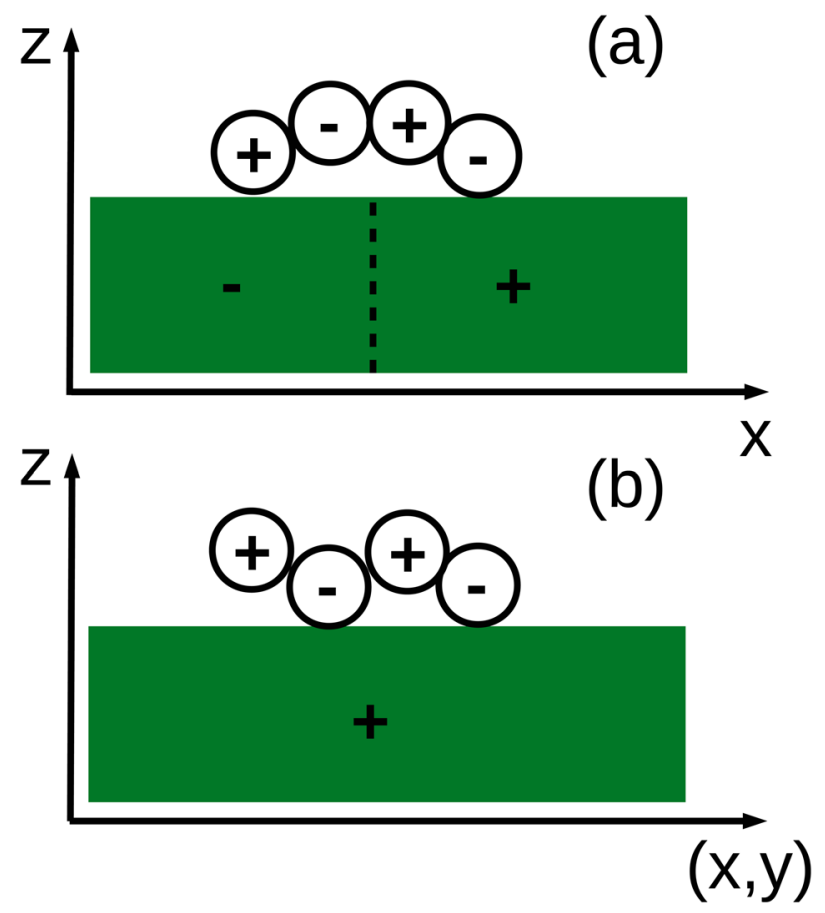

FIG. 7. Representation of configuration of oligomers (a) oriented transversally to the stripes and (b) along the stripes. The electrostatic energy of transverse orientation (a) is lower than parallel orientation (b).
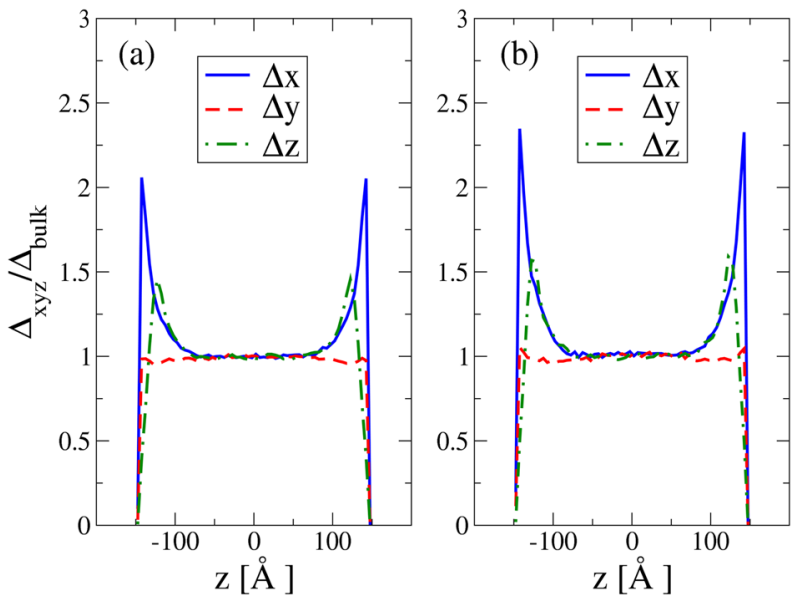

FIG. 8. The $x, y$, and $z$ components of the rms head-to-tail distance for case $n_{x}=1, n_{y}=0$. (a) 20-monomer polyampholytes (periodicity 2-2). (b) 18-monomer polyampholytes (periodicity 3-3).

rms components of the head-to-tail distances, Eq. (9), for 20 and 18-monomer polyampholytes with periodicities 2-2 and 3-3, respectively. Once again we observe a strong elongation of the polyampholyte along the asymmetric $x$ direction. Furthermore, the magnitude of the elongation is proportional to the periodicity of monomers along the polyampholyte backbone.

To better understand the relation between the size of the charged domains and the polyampholyte length, we now study the adsorption for polyampholyte with a different number of monomers $N$. In the bulk, the periodic polyampholytes have a spherical coil shape with the end-to-end distance that scales as $R \sim N^{3 / 5}$. On the other hand, the electrostatic potential produced by the striped surface decays exponentially with distance with decay length $l_{d}=L / 2 \pi n_{x}$. Furthermore, the amplitude of the electrostatic potential in Eq. (7) also decreases with increasing $n_{x}$. We, therefore, expect that when the radius of the polymer coil becomes compatible with $l_{d}$, the adsorption of the polyampholyte will diminish. To check this, we have performed simulations with polyampholytes of different $N$. Unfortunately, difficulty in equilibrating long polyampholyte chains has restricted the system sizes that we were able to explore. Nevertheless, even with the relatively small polymer sizes, we can observe some general trends. To avoid the variation of the amplitude of the electrostatic potential for surfaces with different wavelengths, we will normalize the surface charge density to be $\sigma_{0} / n_{x}=0.1 \mathrm{C} / \mathrm{m}^{2}$. Figure 9 shows that when $R$ becomes comparable to the size of the domains, the adsorption decreases. For polyampholyte with 10 monomers, we find the bulk end-to-end distance to be $R=21 \AA$, which is smaller than the width of the charged domain: 100, 50, 33.3, and $25 \AA$ for $n_{x}=1,2,3$, and 4, respectively. For 18 monomers $R=31 \AA$, which is smaller than the domain width for $n_{x}=1$, 2 , and 3 , but not for $n_{x}=4$. For 30 monomers $R=43 \AA$, which is only smaller than the charged domain size for $n_{x}=1$ and 2 . We observe that the crossover from the increasing to decreasing adsorption happens precisely when the domain size becomes compatible to the width of the charged domains. Under these conditions, 


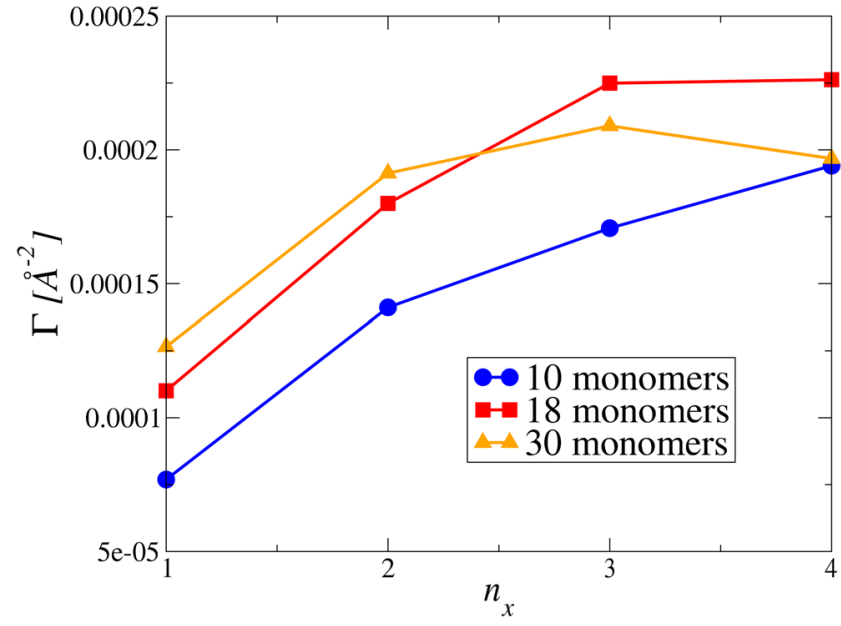

FIG. 9. Adsorption $\Gamma$ for different $n_{x}$. The surface charge density is scaled as $\sigma_{0} / n_{x}=0.1 \mathrm{C} / \mathrm{m}^{2}$ to keep the electrostatic potential at contact constant [see Eq. (7)]. The bulk concentration is adjusted to $\approx 6 \mathrm{mM}$. The polyampholytes have periodicity $1-1$.

entropic, steric repulsion begins to dominate over the electrostatic attraction.

\section{CONCLUSION}

In this paper, we have studied the adsorption of polyampholytes onto charged nanopatterned surfaces using a recently developed Monte Carlo simulation method. ${ }^{31}$ The problem might be relevant for the development of sensors to detect specific proteins. Here, we have explored the adsorption of neutral polyampholytes with periodicities 1-1, 2-2, and 3-3 to striped surfaces of different wavelengths. For the parameters studied, the higher macromolecular adsorption always occurred for the surfaces with the larger charged domains. This could be understood by the preferential adsorption of polyampholytes to the interface between domains of opposite charges. Considering the radius of gyration of the macromolecules, we observed that the polyampholytes adsorb to the surface in a form of an ellipsoid, with the long axis perpendicular to the orientation of the charged stripes. The degree of elongation can be quite significant, increasing with the periodicity of monomer charge distribution along the polyampholyte backbone. The electrostatic potential produced by the nanopatterned surface decreases exponentially with the decay length, which is proportional to the width of charged stripes. We observe that the adsorption first increases with the length of the polyampholytes; however, when the size of the polymer coil becomes comparable with the width of the charged domains, this trend is reversed. At this point, the electrostatic attraction is dominated by the entropic, steric repulsion between the polymer and the wall. It would be interesting to construct a Flory-like theory to try to predict the conformational structure of neutral polyampholytes near a nanopatterned surface. Unfortunately, the presence of many different competing length scales makes this a very challenging task. In the future, we intend to study more realistic larger polyampholytes using molecular dynamics simulations. This will also allow us to explore nonequilibrium effects.

\section{ACKNOWLEDGMENTS}

This work was partially supported by CNPq, INCT-Fcx, and CAPES under Process No. 88882.306664/2013-01 and the US-AFOSR under Grant No. FA9550-12-1-0438.

\section{REFERENCES}

${ }^{1}$ P. Chodanowski and S. Stoll, Macromolecules 34, 2320 (2001).

${ }^{2}$ S. E. Kudaibergenov, Encyclopedia of Polymer Science and Technology (American Cancer Society, 2008).

${ }^{3}$ A. J. Erwin, V. F. Korolovych, Z. Iatridi, C. Tsitsilianis, J. F. Ankner, and V. V. Tsukruk, Macromolecules 51, 4800 (2018).

${ }^{4}$ L. D. Blackman, P. A. Gunatillake, P. Cass, and K. E. S. Locock, Chem. Soc. Rev, 48, 757 (2019).

${ }^{5}$ P. Biehl, M. von der Lühe, S. Dutz, and F. Schacher, Polymers 10, 91 (2018).

${ }^{6}$ K. Morrissey, C. He, R. Chapman, L. Zolnierowski, and M. Stoykovich, Acta Biomater. 40, 192 (2016).

${ }^{7}$ L. R. Denaday, M. V. Miranda, R. M. T. Sánchez, J. M. L. Martínez, L. V. L. Lupano, and V. C. Dall'Orto, Biochem. Eng. J. 58, 57 (2011).

${ }^{8}$ P. Mumick, P. Welch, L. Salazar, and C. McCormick, Macromolecules 27, 323 (1994).

${ }^{9}$ S. E. Kudaibergenov and A. Ciferri, Macromol. Rapid Commun. 28, 1969 (2007).

${ }^{10}$ A. B. Lowe and C. L. McCormick, Chem. Rev. 102, 4177 (2002).

${ }^{11}$ A. Ciferri and S. Kudaibergenov, Macromol. Rapid Commun. 28, 1953 (2007).

${ }^{12}$ M. Vrancken and G. Smets, J. Polym. Sci. 14, 521 (1954).

${ }^{13}$ S. F. Edwards, P. R. King, and P. Pincus, Ferroelectrics 30, 3 (1980).

${ }^{14}$ P. G. Higgs and J. F. Joanny, J. Chem. Phys. 94, 1543 (1991).

${ }^{15}$ Y. Levin and M. C. Barbosa, Europhys. Lett. 31, 513 (1995).

${ }^{16} \mathrm{~J}$. Wittmer, A. Johner, and F. J. Joanny, Europhys. Lett. 24, 263 (1993).

${ }^{17}$ J. M. Victor and J. B. Imbert, Europhys. Lett. 24, 189 (1993).

${ }^{18}$ A. Dobrynin and M. Rubinstein, J. Phys. II 5, 677 (1995).

${ }^{19}$ F. Ozon, J. M. di Meglio, and J. F. Joanny, Eur. Phys. J. E 8, 321 (2002).

${ }^{20}$ A. V. Dobrynin, M. Rubinstein, and J. F. Joanny, Macromolecules 30, 4332 (1997).

${ }^{21}$ A. V. Dobrynin and M. Rubinstein, J. Chem. Phys. 109, 9172 (1998).

${ }^{22}$ M. O. Khan, T. Åkesson, and B. Jönsson, Macromolecules 34, 4216 (2001).

${ }^{23}$ R. R. Netz and D. Andelman, Phys. Rep. 380, 1 (2003).

${ }^{24}$ D. L. Z. Caetano, S. J. De Carvalho, R. Metzler, and A. G. Cherstvy, Phys. Chem. Chem. Phys. 19, 23397 (2017).

${ }^{25}$ M. Muthukumar, J. Chem. Phys. 103, 4723 (1995).

${ }^{26}$ J. McNamara, C. Y. Kong, and M. Muthukumar, J. Chem. Phys. 117, 5354 (2002).

${ }^{27}$ M. Seul and D. Andelman, Science 267, 476 (1995).

${ }^{28}$ R. Parthasarathy, P. A. Cripe, and J. T. Groves, Phys. Rev. Lett. 95, 048101 (2005).

${ }^{29}$ R. D. Piner, J. Zhu, F. Xu, S. Hong, and C. A. Mirkin, Science 283, 661 (1999).

${ }^{30}$ Y. S. Velichko, F. J. Solis, and M. O. de la Cruz, J. Chem. Phys. 128, 144706 (2008).

${ }^{31}$ A. Bakhshandeh, A. P. dos Santos, and Y. Levin, Soft Matter 14, 4081 (2018).

${ }^{32}$ R. M. Adar, D. Andelman, and H. Diamant, Adv. Colloid Interface 247, 198 (2017).

${ }^{33}$ T. Wallin and P. Linse, Langmuir 12, 305 (1996).

${ }^{34} \mathrm{~T}$. Wallin and P. Linse, J. Phys. Chem. B 100, 17873 (1996).

${ }^{35} \mathrm{~T}$. Wallin and P. Linse, J. Phys. Chem. B 101, 5506 (1997).

${ }^{36}$ A. P. dos Santos, M. Girotto, and Y. Levin, J. Phys. Chem. B 120, 10387 (2016).

${ }^{37}$ A. P. dos Santos, M. Girotto, and Y. Levin, J. Chem. Phys. 144, 144103 (2016).

${ }^{38}$ B. Smith and D. Frenkel, Understanding Molecular Simulations (Academic, New York, 1996).

${ }^{39}$ M. P. Allen and D. J. Tildesley, Computer Simulation of Liquids (Oxford University Press, Oxford, 1987).

${ }^{40}$ I.-C. Yeh and M. L. Berkowitz, J. Chem. Phys. 111, 3155 (1999). 